\title{
Modelagem e análise de um sistema de controle automático da altura de corte em colhedoras ${ }^{1}$
}

\author{
Leidy Z. L. Rafull2 ${ }^{2}$ Daniel M. de Queiroz ${ }^{3}$, Cristiano M. A. de Souza ${ }^{2}$ \& Francisco de A. de C. Pinto ${ }^{3}$
}

\begin{abstract}
RESUMO
Neste estudo, desenvolveu-se um modelo de simulação para analisar a dinâmica de um sistema a fim de controlar automaticamente a altura de corte em colhedoras. Este modelo foi implementado com base nas equações diferenciais, que descrevem a dinâmica da plataforma de corte, o comportamento do sistema hidráulico e o controlador. O sistema de controle realimentado simulado foi constituído de uma célula de carga, destinada a medir a força de contato entre o solo e o mecanismo de corte, um controlador PID e um sistema hidráulico de atuação, composto de uma válvula proporcional direcional de quatro vias e um atuador. Analisou-se a influência da velocidade de deslocamento da máquina $\left(1,0,1,5\right.$ e 2,0 m s$\left.~^{-1}\right)$, da pressão de suprimento do sistema hidráulico $(6,8,13,7$ e 20,6 MPa), da massa da plataforma $(40,60$ e $80 \mathrm{~kg})$ e da força de contato de referência do controlador (200, 400 e $800 \mathrm{~N})$ no desempenho do sistema de controle. Os aumentos na pressão de suprimento, massa da plataforma e força de referência, proporcionaram melhores condições para o funcionamento do sistema de controle proposto.
\end{abstract}

Palavras-chave: simulação, controlador PID, válvula proporcional de 4 vias

\section{Modeling and analysis of an automatic control system for harvesting machine cutting height}

\begin{abstract}
A simulation model to analyze the dynamics of a system to control automatically the cutting height in harvesters was developed. The model was developed using the differential equations that describe the dynamics of the cuttig platform, the behavior of the hydraulic system and the controller. The simulated feedback control system was composed by a load cell that measures the contact force between the soil and the cutting device, a feedback controller (PID) and a hydraulic system with proportional directional four-way valve and actuator. The effect of the following variables in the controller performance was analyzed: speed of the cutting device $\left(1.0,1.5\right.$ and $\left.2.0 \mathrm{~m} \mathrm{~s}^{-1}\right)$; pressure in the supply line of the hydraulic system (6.8, 13.7 and 20.6 MPa); ballast added in the cutting mechanism (40, 60 and $80 \mathrm{~kg}$ ) and the contact force between the cutting device and the soil $(200,400$ and $800 \mathrm{~N})$. The developed model showed to be a useful tool for analyzing the dynamics of the controller. The increase in the supply pressure, mass of the cut platform and reference force provided better performance of the control system.
\end{abstract}

key words: simulation, PID controller, proportional four-way valve

1 Parte da Tese de Doutorado da primeira autora, apresentada à UFV

2 FCA/UFGD. CEP 79804-970, Dourados, MG. Fone: (67) 3411 3841. E-mail: zulys@ufgd.edu.br; csouza@ufgd.edu.br

${ }_{3}^{3}$ DEA/UFV. CEP. 36570-000. Viçosa, MG. Fone: (31) 3899 2783. E-mail: queiroz@ufv.br; facpinto@ufv.br 


\section{INTRODUÇÃO}

A utilização de máquinas no processo de colheita reduziu notavelmente o tempo necessário para execução desta operação, mas humanizou o trabalho do homem no campo. Apesar dessas vantagens, os mecanismos de corte das colhedoras apresentam a dificuldade em se acompanhar, de maneira eficiente, o perfil do terreno, podendo favorecer a realização de um corte acima da altura ótima ou, até mesmo, o enterramento desses mecanismos. Esses fatos provocam perdas, presença de impurezas no material colhido, desgaste excessivo das lâminas de corte, sobrecarga dos elementos de transmissão, aumento da exigência de potência e, no caso da cana-de-açúcar, danos às soqueiras.

Em decorrência das imperfeições provocadas por este processo, Gómez (1996) determinou perdas variando de 6,7 a $15,7 \mathrm{t} \mathrm{ha}^{-1}$ em canaviais com produtividade média de $70 \mathrm{t} \mathrm{ha}^{-1}$ e, na colheita do feijão, Souza et al. (2001) verificaram que $50 \%$ das perdas totais de grãos ocorreram na plataforma da colhedora.

O uso de sistemas de controle automático de posição dos mecanismos de corte é indicado para melhorar a eficiência das colhedoras (Rafull et al., 2004). Aponta-se também, como vantagem, a redução da fadiga do operador e a diminuição dos danos ocasionados nos mecanismos da máquina, além de proporcionar um produto final de melhor qualidade.

Existem modelos de colhedoras dotadas com sistemas automáticos de posição do mecanismo de corte, nos quais têm sido utilizados controladores de duas posições e atuadores hidráulicos comandados por válvulas direcionais de três vias. Este sistema, embora simples e de baixo custo, apresenta comprometimento no acompanhamento do perfil do terreno, devido à impossibilidade de regulagem do fluxo de óleo que alimenta o atuador. O uso de válvulas hidráulicas de fluxo e de pressão variáveis, apresenta-se como opção à implementação de sistemas de controle proporcional.

O desenvolvimento de modelos matemáticos, considerando-se as características do sistema hidráulico de atuação e a geometria da plataforma de corte, apresenta-se como ferramenta útil para caracterização da dinâmica dos sistemas de controle. O uso das técnicas de modelagem e simulação facilita o estudo do comportamento do sistema, em virtude da criação de cenários que reproduzem as características do sistema real. Deste modo, é possível se reduzir custos por meio de análise e revelar a integridade e viabilidade de determinado projeto, em termos técnicos e econômicos (Winston, 1994).

Portanto, com o presente trabalho objetivou-se apresentar uma análise utilizando-se um modelo de simulação de um sistema proposto para controlar automaticamente a posição do mecanismo de corte em plataformas de colhedoras, visando à melhoria do processo de colheita. Este trabalho propõe um sistema de controle de posição automático, formado por uma célula de carga, uma válvula proporcional direcional de quatro vias e um controlador do tipo proporcional-integralderivativo, buscando favorecer o acompanhamento do perfil do solo.

\section{MATERIAL E MÉTODOS}

O trabalho foi desenvolvido no Laboratório de Projetos de Máquinas e Visão Artificial do Departamento de Engenharia Agrícola da Universidade Federal de Viçosa. O sistema de controle idealizado foi formado por uma célula de carga para medir a força de contato entre o solo e o mecanismo de corte, um controlador que, após comparar o sinal medido pelo transdutor com a força de contato de referência, emite um sinal elétrico de correção do erro, que é recebido pelo sistema de atuação hidráulico, encarregado de posicionar o mecanismo de corte na altura desejada.

A plataforma de corte modelada foi idealizada a partir daquela apresentada por Lopes (2000). Realizaram-se algumas modificações no projeto original, ou seja, o sensor de altura foi substituído por uma célula de carga e o ponto de conexão do cilindro hidráulico foi reposicionado, Figura 1. A plataforma é articulada no ponto $\mathrm{C}$ e, em sua parte frontal, está posicionada a célula de carga. Um lastro representa o peso da plataforma e o ponto $\mathrm{F}$, a extremidade da ponta esférica ligada à célula de carga. Sua posição horizontal e vertical varia com o deslocamento da máquina, considerado constante, e as irregularidades do perfil do solo, Eqs. 1 e 2.

$$
\begin{gathered}
\mathrm{x}_{\mathrm{F}}=\left(\mathrm{x}_{1}+\mathrm{x}_{5}\right) \operatorname{sen} \theta+\mathrm{x}_{9} \operatorname{sen}\left(\theta_{3}+\theta-\theta_{0}\right)+\dot{\mathrm{x}} \mathrm{t} \\
\mathrm{y}_{\mathrm{F}}=\left[\mathrm{x}_{6}+\left(\mathrm{x}_{1}+\mathrm{x}_{5}\right) \cos \theta_{0}\right]-\left[\mathrm{x}_{6}+\left(\mathrm{x}_{1}+\mathrm{x}_{5}\right) \cos \theta\right]
\end{gathered}
$$

em que:

$\mathrm{X}_{\mathrm{F}}$ - coordenada da ponta esférica ligada à célula de carga no eixo $\mathrm{x}, \mathrm{m}$

$\mathrm{y}_{\mathrm{F}}$ - coordenada da ponta esférica ligada à célula de carga no eixo y, m

$\mathrm{x}_{1}$ - distância entre os pontos $\mathrm{C}$ e $\mathrm{A}, \mathrm{m}$

$\mathrm{x}_{5}$ - distância entre os pontos A e D, m

$\theta$ - ângulo formado entre as barras BC e CD, rad

$\theta_{0}-$ valor inicial do ângulo $\theta$, rad

$\mathrm{x}_{6}$ - distância entre o ponto $\mathrm{F}$ e o ponto $\mathrm{E}$, $\mathrm{m}$

$\dot{\mathrm{x}}$ - velocidade de deslocamento da máquina, $\mathrm{m} \mathrm{s}^{-1}$

$\mathrm{t}$ - tempo, $\mathrm{s}$

$\mathrm{q}_{3}$ - ângulo formado entre a linha imaginária que passa pelos pontos $\mathrm{F}$ e D, e a linha perpendicular ao segmento DE, rad

$\mathrm{X}_{9}$ - distância entre os pontos D e F, m

$\mathrm{O}$ ângulo $\mathrm{q}_{3}$ foi determinado empregando-se a expressão:

$$
\theta_{3}=\frac{\pi}{2}-\arctan \left(\frac{\mathrm{x}_{6}}{\mathrm{x}_{7}}\right)
$$

em que:

$\mathrm{x}_{7}$ - comprimento da barra ED, $\mathrm{m}$

A distância entre o ponto D e o ponto $\mathrm{F}\left(\mathrm{x}_{9}\right)$ foi determinada utilizando-se a expressão:

$$
\mathrm{x}_{9}=\sqrt{\left(\mathrm{x}_{6}\right)^{2}+\left(\mathrm{x}_{7}\right)^{2}}
$$

O ângulo formado entre a barra CB e a linha imaginária, 


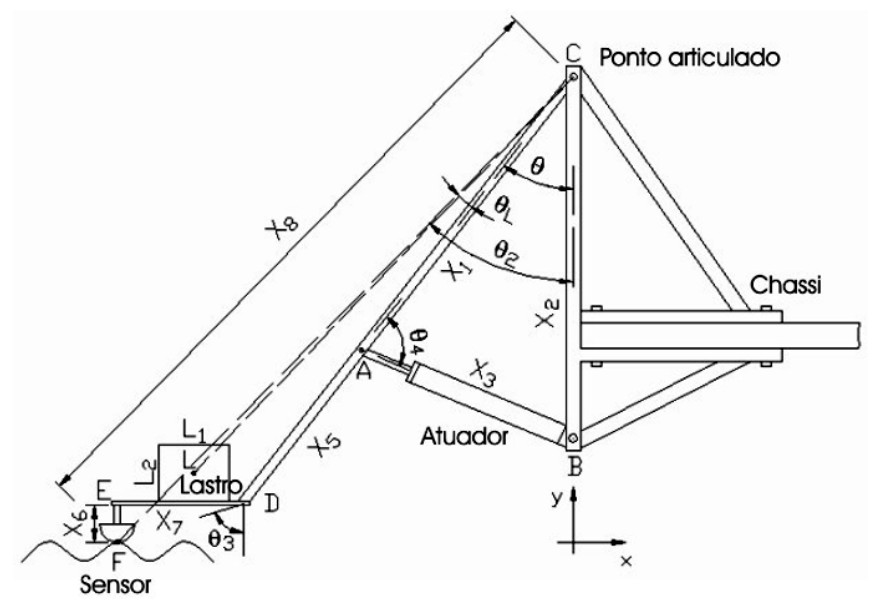

Figura 1. Esquema da plataforma de corte

que passa pelos pontos $\mathrm{C}$ e $\mathrm{F}$, foi determinado por meio da expressão:

$$
\theta_{2}=\operatorname{arcsen}\left(\frac{x_{F}-\dot{x} t}{x_{8}}\right)
$$

em que:

$\theta_{2}$ - ângulo formado entre a barra CB e a linha imaginária, que passa pelos pontos $\mathrm{C}$ e $\mathrm{F}$, rad $\mathrm{x}_{8}$ - distância entre os pontos C e F, m

A distância entre os pontos $\mathrm{C}$ e $\mathrm{F}$ foi determinada como:

$$
\mathrm{x}_{8}=\sqrt{\left(\mathrm{x}_{\mathrm{F}}-\dot{\mathrm{xt}}\right)^{2}+\left[\mathrm{y}_{\mathrm{F}}+\mathrm{x}_{6}+\left(\mathrm{x}_{1}+\mathrm{x}_{5}\right) \cos \theta\right]^{2}}
$$

Quando a ponta esférica ligada à célula de carga entra em contato com o solo, gera-se uma força calculada pela expressão:

$$
\mathrm{F}_{\mathrm{C}}=-\mathrm{K}_{\mathrm{s}}\left(\mathrm{y}_{\mathrm{F}}-\mathrm{y}_{\mathrm{s}}\right)-\mathrm{C}_{\mathrm{s}} \dot{\mathrm{y}}_{\mathrm{F}}
$$

em que:

$\mathrm{F}_{\mathrm{C}}$ - força de contato entre a ponta esférica ligada à célula de carga e o solo, $\mathrm{N}$

$\mathrm{C}_{\mathrm{s}}$ - constante de amortecimento do solo, $\mathrm{Ns} \mathrm{m}^{-1}$

$\mathrm{K}_{\mathrm{s}}$ - constante elástica do solo, $\mathrm{N} \mathrm{m}^{-1}$

$\mathrm{y}_{\mathrm{s}}-$ altura do perfil do solo, $\mathrm{m}$

$\dot{\mathrm{y}}_{\mathrm{F}}$ - velocidade de penetração da ponta no solo, $\mathrm{m} \mathrm{s}^{-1}$

A velocidade de penetração da ponta no solo foi determinada por:

$$
\dot{\mathrm{y}}_{\mathrm{F}}=\dot{\theta} \mathrm{x}_{8} \cos \left(\frac{\pi}{2}-\theta_{2}\right)
$$

em que:

$\dot{\theta}$ - velocidade angular da plataforma, rad s ${ }^{-1}$

O valor da constante de amortecimento foi admitido como dependente da profundidade de penetração da ponta esférica ligada à célula de carga no solo, uma vez que a capacidade amortecedora do solo varia com a profundidade. Para a situação em que não existe penetração, a constante de amor- tecimento e a constante elástica foram consideradas iguais a zero. Para penetração maior ou igual a $0,01 \mathrm{~m}$, admitiu-se que a constante assumiu o valor máximo de $1000 \mathrm{Ns} \mathrm{m}^{-1}$. Para se obter valores intermediários da constante de amortecimento, utilizou-se uma função de interpolação de terceiro grau, Eq. 9, que pode ser usada para representar comportamento de sistemas, onde há choque (MDI, 1993), como é o caso do contato entre a ponta esférica e o solo.

$$
\mathrm{C}_{\mathrm{S}}=\mathrm{C}_{\mathrm{S} \min }+\left(\mathrm{C}_{\mathrm{S}_{\max }}-\mathrm{C}_{\mathrm{S} \min }\right)\left[3-2\left(\frac{\mathrm{y}-\mathrm{y}_{0}}{\mathrm{y}_{1}-\mathrm{y}_{0}}\right)\right] \frac{\left(\mathrm{y}-\mathrm{y}_{0}\right)^{2}}{\left(\mathrm{y}_{1}-\mathrm{y}_{0}\right)^{2}}
$$

em que:

$\mathrm{C}_{\mathrm{S}}$ - constante de amortecimento, $\mathrm{Ns} \mathrm{m}^{-1}$

$\mathrm{C}_{\mathrm{Smax}}$ - valor máximo da função passo, $\mathrm{Ns} \mathrm{m}^{-1}$

$\mathrm{C}_{\text {Smin }}$ - valor mínimo da função passo, $\mathrm{Ns} \mathrm{m}^{-1}$

y - penetração da ponta esférica da célula de carga, $m$

$\mathrm{y}_{0}$ - valor no qual $\mathrm{C}_{\mathrm{S}}$ é igual ao valor mínimo, Ns $\mathrm{m}^{-1}$

$\mathrm{y}_{1}$ - valor no qual $\mathrm{C}_{\mathrm{S}}$ passa a ser constante e igual a $\mathrm{C}_{\text {Smax }}$, Ns m ${ }^{-1}$

O perfil do solo foi admitido como representado por uma senóide e, assim, a altura do perfil do solo foi calculada por meio da expressão:

$$
y_{S}=a \operatorname{sen}\left(\frac{2 \pi x_{F}}{x_{\text {per }}}\right)
$$

em que:

$\mathrm{a}$ - amplitude das ondas senoidais do solo, $\mathrm{m}$

$\mathrm{x}_{\text {per }}$ - comprimento das ondas senoidais do solo, $\mathrm{m}$

Com base na segunda lei de Newton obteve-se a equação diferencial que descreve o movimento angular da plataforma de corte.

$$
\mathrm{I}_{\mathrm{xx}} \ddot{\theta}=\tau_{1}+\tau_{2}+\tau_{3}
$$

em que:

$\mathrm{I}_{\mathrm{Xx}}$ - momento de inércia da plataforma, $\mathrm{kg} \mathrm{m}^{2}$

$\ddot{\theta}$ - aceleração angular da plataforma, rad s$^{-2}$

$\tau_{1}$ - torque produzido pela força do atuador relativo ao ponto $\mathrm{C}$, $\mathrm{Nm}$

$\tau_{2}$ - torque produzido pela força do solo relativo ao ponto C, Nm

$\tau_{3}$ - torque produzido pelo peso da estrutura e o peso dos lastros, relativo ao ponto $\mathrm{C}, \mathrm{Nm}$

A Eq. 11 foi transformada nas duas equações diferenciais de primeira ordem, a seguir:

$$
\begin{gathered}
\dot{\theta}=\omega \\
\dot{\omega}=\frac{1}{\mathrm{I}_{\mathrm{xx}}}\left(\tau_{1}+\tau_{2}+\tau_{3}\right)
\end{gathered}
$$

em que:

$\omega$ - velocidade angular da plataforma, $\operatorname{rad~s}^{-1}$

$\dot{\omega}$ - aceleração angular da plataforma, $\operatorname{rad~s}^{-2}$

O torque produzido pela força do atuador no ponto de 
articulação da plataforma (C) foi determinado pela expressão:

$$
\tau_{1}=\mathrm{F}_{\mathrm{a}} \mathrm{x}_{1} \operatorname{sen} \theta_{4}
$$

em que:

$F_{a}$ - força produzida pelo atuador para movimentar a plataforma de corte, $\mathrm{N}$

$\theta_{4}$ - ângulo formado entre a barra CD e o segmento $\mathrm{AB}$, rad

A força produzida pelo atuador para movimentar a plataforma de corte foi determinada em função do sistema hidráulico utilizado e o ângulo $\theta_{4}$ o foi pela expressão:

$$
\theta_{4}=\arccos \left[\frac{\left(\mathrm{x}_{2}^{2}-\mathrm{x}_{3}^{2}-\mathrm{x}_{1}^{2}\right)}{-2 \mathrm{x}_{3} \mathrm{x}_{1}}\right]
$$

em que:

$\mathrm{x}_{2}$ - distância entre o ponto de apoio da base do atuador (B) e o ponto de articulação da plataforma $(\mathrm{C}), \mathrm{m}$

$\mathrm{x}_{3}$ - distância entre os pontos $\mathrm{A}$ e $\mathrm{B}, \mathrm{m}$

A distância $x_{3}$ foi determinada através da expressão:

$$
\mathrm{x}_{3}=\sqrt{\left(\mathrm{x}_{1} \operatorname{sen} \theta\right)^{2}+\left(\mathrm{x}_{2}-\mathrm{x}_{1} \cos \theta\right)^{2}}
$$

O torque que a força de contato da ponta esférica ligada à célula de carga e o solo produz, no ponto de articulação da plataforma, foi calculado como:

$$
\tau_{2}=\mathrm{F}_{\mathrm{C}}\left[\left(\mathrm{x}_{1}+\mathrm{x}_{5}\right) \operatorname{sen} \theta+\mathrm{x}_{9} \operatorname{sen}\left(\theta+\theta_{3}-\theta_{0}\right)\right]
$$

O torque produzido pelo peso da estrutura e o peso dos lastros no ponto de articulação foi determinado pela expressão:

$$
\tau_{3}=-\left[M_{B} g\left(\frac{x_{1}+x_{5}}{2}\right) \operatorname{sen} \theta+M_{L} g r_{L} \operatorname{sen}\left(\theta+\theta_{L}\right)\right]
$$

em que:

$\mathrm{M}_{\mathrm{B}}$ - massa da barra CD, kg

$\mathrm{M}_{\mathrm{L}}$ - massa do lastro da plataforma, $\mathrm{kg}$

$\mathrm{g}$ - aceleração da gravidade, $\mathrm{m} \mathrm{s}^{-2}$

$\mathrm{r}_{\mathrm{L}}$ - distância entre os pontos $\mathrm{L}$ e de articulação da plataforma (C), m

$\theta_{\mathrm{L}}$ - ângulo formado entre a barra CD e a linha imaginária que passa pelos pontos $\mathrm{L}$ e $\mathrm{C}$, rad

O ângulo $\theta_{\mathrm{L}}$ e a distância $\mathrm{r}_{\mathrm{L}}$ foram determinados através das expressões:

$$
\begin{gathered}
\theta_{\mathrm{L}}=\arctan \left(\frac{\mathrm{x}_{\mathrm{L}}}{\mathrm{y}_{\mathrm{L}}}\right)-\theta \\
\mathrm{r}_{\mathrm{L}}=\sqrt{\mathrm{x}_{\mathrm{L}}{ }^{2}+\mathrm{y}_{\mathrm{L}}^{2}}
\end{gathered}
$$

em que:

$\mathrm{x}_{\mathrm{L}}$ - coordenada do centro dos lastros no eixo $\mathrm{x}, \mathrm{m}$

$\mathrm{y}_{\mathrm{L}}$ - coordenada do centro dos lastros no eixo $\mathrm{y}, \mathrm{m}$ As coordenadas $\mathrm{x}_{\mathrm{L}}$ e $\mathrm{y}_{\mathrm{L}}$ são dadas por:

$$
\begin{aligned}
& \mathrm{x}_{\mathrm{L}}=\left(\mathrm{x}_{1}+\mathrm{x}_{5}\right) \operatorname{sen} \theta+\frac{\mathrm{L}_{1}}{2} \\
& \mathrm{y}_{\mathrm{L}}=\left(\mathrm{x}_{1}+\mathrm{x}_{5}\right) \cos \theta-\frac{\mathrm{L}_{2}}{2}
\end{aligned}
$$

em que:

$$
\begin{aligned}
& \mathrm{L}_{1} \text { - largura do lastro, } \mathrm{m} \\
& \mathrm{L}_{2} \text { - altura do lastro, } \mathrm{m}
\end{aligned}
$$

As características geométricas utilizadas foram semelhantes às usadas por Lopes (2000) e os valores de $K_{S}$ e $C_{S}$ determinados em função da profundidade de penetração da célula de carga no solo, Tabela 1.

Tabela 1. Dimensões e parâmetros usados no modelo do mecanismo de corte

\begin{tabular}{cccc}
\hline Parâmetro & Valor & Parâmetro & Valor \\
$\mathrm{X}_{1}$ & $0,631 \mathrm{~m}$ & $\theta_{0}$ & $0,78 \mathrm{rad}$ \\
$\mathrm{x}_{2}$ & $0,615 \mathrm{~m}$ & $\mathrm{~K}_{\mathrm{S}}$ & $10000 \mathrm{~N} \mathrm{~m}^{-1}$ \\
$\mathrm{X}_{3 \min }$ & $0,370 \mathrm{~m}$ & $\mathrm{C}_{\text {smax }}$ & $1000 \mathrm{Ns} \mathrm{m}^{-1}$ \\
$\mathrm{X}_{5}$ & $0,369 \mathrm{~m}$ & $\mathrm{C}_{\text {smin }}$ & $0 \mathrm{Ns} \mathrm{m}^{-1}$ \\
$\mathrm{X}_{6}$ & $0,105 \mathrm{~m}$ & $\mathrm{y}_{0}$ & 0 \\
$\mathrm{X}_{7}$ & $0,125 \mathrm{~m}$ & $\mathrm{y}_{1}$ & $0,01 \mathrm{~m}$ \\
$\mathrm{M}_{\mathrm{B}}$ & $3,14 \mathrm{~kg}$ & & \\
\hline
\end{tabular}

O controlador proporcional-integral-derivativo foi projetado com vistas a minimizar o erro verificado entre uma força de referência e a força de contato, Eq. 23, medida pela célula de carga. Este erro foi corrigido pelo deslocamento do carretel da válvula hidráulica.

$$
\mathrm{e}_{\mathrm{s}}=\mathrm{F}_{\mathrm{C}}-\mathrm{F}_{\mathrm{R}}
$$

em que:

$F_{R}$ - força de contato de referência do controlador, $N$

O sinal de controle do controlador PID, segundo Ogata (1985), pode ser dado pela expressão:

$$
u=K_{p} e_{s}(t)+K_{i} \int e_{s}(t) d t+K_{d}\left[\frac{d e_{s}(t)}{d t}\right]
$$

em que:

$$
\begin{aligned}
\mathrm{u} & - \text { sinal de controle } \\
\mathrm{K}_{\mathrm{P}} & - \text { constante proporcional } \\
\mathrm{e}_{\mathrm{s}} & - \text { sinal de erro } \\
\mathrm{t} & - \text { tempo } \\
\mathrm{K}_{\mathrm{i}} & - \text { constante de integração } \\
\mathrm{K}_{\mathrm{d}} & - \text { constante de derivação }
\end{aligned}
$$

Os valores das constantes do controlador $\left(K_{\mathrm{P}}, \mathrm{K}_{\mathrm{i}}, \mathrm{K}_{\mathrm{d}}\right)$ do sistema foram obtidos por meio de algoritmo numérico, que os variaram até obter um mínimo erro na determinação da força de contato. O sistema hidráulico de atuação, encarregado de posicionar a plataforma de corte, foi composto por um cilindro hidráulico e uma válvula direcional proporcional, de quatro vias. O cilindro hidráulico é do tipo diferencial de dupla ação, com um curso do pistão de 0,21 m e áreas da cabeça e da coroa do pistão de 5,07 x $10^{-4}$ e $3,01 \times 10^{-4} \mathrm{~m}^{2}$, respectivamente. A válvula de controle é de carretel deslizante e 
o deslocamento do carretel da válvula é proporcional ao sinal elétrico de alimentação, enviado pelo controlador.

O modelo matemático que caracteriza a dinâmica do sistema hidráulico, formado por uma válvula proporcional de quatro vias controlando um cilindro diferencial de dupla ação, Figura 2, foi derivado da equação de continuidade para cada câmara (Merrit, 1967). As duas equações diferenciais que descrevem o comportamento do sistema, são:

$$
\begin{gathered}
Q_{1}-C_{\text {ip }}\left(P_{1}-P_{2}\right)-C_{\text {ep }} P_{1}=\frac{d V_{1}}{d t}+\frac{V_{1}}{\beta_{e}} \frac{d P_{1}}{d t} \\
C_{\text {ip }}\left(P_{1}-P_{2}\right)-C_{\text {ep }} P_{2}-Q_{2}=\frac{d V_{2}}{d t}+\frac{V_{2}}{\beta_{e}} \frac{d P_{2}}{d t}
\end{gathered}
$$

em que:

$\mathrm{Q}_{1}$ - vazão na câmara de levantamento, $\mathrm{m}^{3} \mathrm{~s}^{-1}$

$\mathrm{Q}_{2}$ - vazão na câmara de retrocesso, $\mathrm{m}^{3} \mathrm{~s}^{-1}$

$\mathrm{P}_{1}$ - pressão na câmara de levantamento do atuador, $\mathrm{Pa}$

$\mathrm{P}_{2}$ - pressão na câmara de retrocesso do atuador, $\mathrm{Pa}$

$\mathrm{V}_{1}$ - volume na câmara de levantamento, $\mathrm{m}^{3}$

$\mathrm{V}_{2}$ - volume na câmara de retrocesso, $\mathrm{m}^{3}$

$\mathrm{C}_{\mathrm{ip}}$ - coeficiente de vazamento interno do pistão, $\mathrm{m}^{3} \mathrm{~s}^{-1} \mathrm{~Pa}^{-1}$

$\mathrm{C}_{\text {ep }}$ - coeficiente de vazamento externo do pistão, $\mathrm{m}^{3} \mathrm{~s}^{-1} \mathrm{~Pa}^{-1}$

$\beta_{\mathrm{e}}-$ módulo efetivo do sistema, $\mathrm{Pa}$

$\mathrm{t}$ - tempo, $\mathrm{s}$

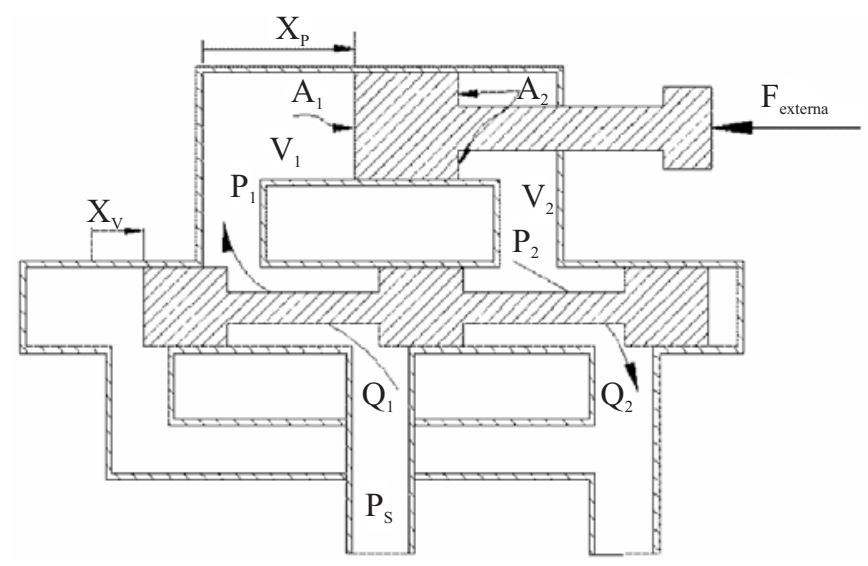

Figura 2. Válvula direcional proporcional de quatro vias controlando pistão em condição de avanço do atuador proposta por Lopes (2000) com modificações

Os volumes das câmaras de levantamento e retrocesso foram determinados pelas expressões:

$$
\begin{aligned}
& V_{1}=V_{01}+A_{1} x_{p} \\
& V_{2}=V_{02}-A_{2} x_{p}
\end{aligned}
$$

em que:

$\mathrm{V}_{01}$ - volume inicial na câmara de levantamento, $\mathrm{m}^{3}$

$\mathrm{V}_{02}$ - volume inicial na câmara de retrocesso, $\mathrm{m}^{3}$

$\mathrm{A}_{1}$ - área da cabeça do pistão do atuador, $\mathrm{m}^{2}$

$\mathrm{A}_{2}$ - área da coroa do pistão do atuador, $\mathrm{m}^{2}$

$\mathrm{x}_{\mathrm{p}}$ - deslocamento da haste do cilindro, $\mathrm{m}$
O deslocamento da haste do cilindro hidráulico foi determinado pela expressão:

$$
\mathrm{x}_{\mathrm{P}}=\mathrm{x}_{3}-\mathrm{x}_{3 \min }
$$

em que:

$\mathrm{x}_{3 \min }$ - valor mínimo do comprimento do braço que suporta o atuador, quando o cilindro está recolhido, $\mathrm{m}$

A vazão no sistema foi calculada considerando-se a magnitude do deslocamento do carretel da válvula e da pressão em cada câmara. Quando o deslocamento do carretel da válvula era positivo $\left(\mathrm{x}_{\mathrm{V}}>0\right)$ e a pressão na câmara de levantamento menor que a pressão de suprimento $\left(\mathrm{P}_{1}<\mathrm{P}_{\mathrm{S}}\right)$, a vazão na câmara de levantamento foi calculada pela expressão:

$$
\mathrm{Q}_{1}=\mathrm{C}_{\mathrm{d}}\left(\mathrm{wx}_{\mathrm{V}}\right) \sqrt{\frac{2}{\rho}\left(\mathrm{P}_{\mathrm{S}}-\mathrm{P}_{1}\right)}
$$

em que:

$\mathrm{C}_{\mathrm{d}}$ - coeficiente de descarga, adimensional

$\mathrm{w}$ - gradiente da área do orifício principal, $\mathrm{m}^{2} \mathrm{~m}^{-1}$

$\mathrm{P}_{\mathrm{S}}$ - pressão de suprimento do sistema hidráulico, $\mathrm{Pa}$

$\rho$ - massa específica do fluido hidráulico, $\mathrm{kg} \mathrm{m}^{-3}$

Quando a pressão na câmara de levantamento era igual à pressão de suprimento $\left(\mathrm{P}_{1}=\mathrm{P}_{\mathrm{S}}\right), \mathrm{Q}_{1}$ foi assumido como sendo igual a zero. No caso em que foi mantido o valor positivo do deslocamento do carretel e a pressão na câmara de retrocesso foi maior que zero $\left(\mathrm{P}_{2}>0\right)$, a vazão na câmara de retrocesso foi calculada pela expressão:

$$
\mathrm{Q}_{2}=-\mathrm{C}_{\mathrm{d}}\left(\mathrm{wx}_{\mathrm{V}}\right) \sqrt{\frac{2}{\rho}\left(\mathrm{P}_{2}\right)}
$$

Caso contrário, $\mathrm{Q}_{2}$ foi considerada igual a zero. Quando o deslocamento do carretel da válvula foi negativo $\left(\mathrm{x}_{\mathrm{V}}<0\right)$ e a pressão na câmara de levantamento foi maior que zero $\left(\mathrm{P}_{1}>0\right)$, a vazão na câmara de levantamento foi calculada pela expressão:

$$
\mathrm{Q}_{1}=\mathrm{C}_{\mathrm{d}}\left(\mathrm{wx}_{\mathrm{V}}\right) \sqrt{\frac{2}{\rho}\left(\mathrm{P}_{1}\right)}
$$

Em não satisfazendo essas condições, admitiu-se $\mathrm{Q}_{1}$ como sendo igual a zero. No caso em que o deslocamento do carretel da válvula foi negativo e a pressão na câmara de retrocesso menor que a pressão de suprimento $\left(\mathrm{P}_{2}<\mathrm{P}_{\mathrm{s}}\right)$, a vazão na câmara de retrocesso foi calculada pela expressão:

$$
\mathrm{Q}_{2}=-\mathrm{C}_{\mathrm{d}}\left(\mathrm{wx}_{\mathrm{V}}\right) \sqrt{\frac{2}{\rho}\left(\mathrm{P}_{\mathrm{S}}-\mathrm{P}_{2}\right)}
$$

Caso contrário, $\mathrm{Q}_{2}$ foi considerada igual a zero. A força produzida pelo atuador, para movimentar a plataforma de corte, foi determinada pela expressão:

$$
\mathrm{F}_{\mathrm{a}}=\mathrm{P}_{1} \mathrm{~A}_{1}-\mathrm{P}_{2} \mathrm{~A}_{2}
$$


Foram estabelecidas, como condições iniciais, um deslocamento do carretel da válvula de 1,0 x $10^{-7} \mathrm{~m}$ (um valor positivo e diferente de zero), um ângulo do braço da plataforma com a vertical de 0,78 radiano e uma velocidade angular do braço igual a zero. Também se estabeleceu uma pressão inicial na câmara de levantamento igual a 75\% do valor da pressão de suprimento máxima e uma pressão de retorno de $1 \mathrm{~Pa}$. A solução do sistema de equações diferenciais composto pelas Eqs. 12, 13, 25 e 26 foi obtida com o método de Runge Kutta de $4^{\text {a }}$ ordem (Press et al., 1992). Os valores das constantes usadas na modelagem do sistema hidráulico foram obtidos de Merrit (1967) conforme Tabela 2.

Tabela 2. Parâmetros do sistema hidráulico de atuação

\begin{tabular}{cc}
\hline Parâmetro & Valor \\
$C d$ & 0,61 \\
$\rho$ & $800 \mathrm{~kg} \mathrm{~m}^{-3}$ \\
$\beta$ & $689,4 \mathrm{MPa}$ \\
$W$ & $7,9 \times 10^{-5} \mathrm{~m}^{2} \mathrm{~m}^{-1}$ \\
$\mathrm{C}_{\mathrm{ep}}$ & $5,0 \times 10^{-8} \mathrm{~m}^{3} \mathrm{~s}^{-1} \mathrm{~Pa}^{-1}$ \\
$\mathrm{C}_{\text {ip }}$ & $5,0 \times 10^{-8} \mathrm{~m}^{3} \mathrm{~s}^{-1} \mathrm{~Pa}^{-1}$ \\
\hline
\end{tabular}

Para simular o comportamento do sistema de controle, implementou-se um programa computacional utilizando-se o modelo matemático desenvolvido e a linguagem de programação Fortran. Estudou-se a influência que parâmetros como a velocidade de deslocamento (1,0, 1,5 e 2,0 m s$\left.{ }^{-1}\right)$, a pressão de suprimento do sistema hidráulico $(6,8,13,7$ e 20,6 MPa), a massa da plataforma de corte (40, 60 e $80 \mathrm{~kg}$ ) e a força de contato de referência do controlador (200, 400 e $800 \mathrm{~N}$ ) exercem na dinâmica do sistema, simulados com duração de $5 \mathrm{~s}$ e incremento de $10^{-3} \mathrm{~s}$.

O solo foi considerado função senoidal com amplitude de $0,05 \mathrm{~m}$ e comprimento de ondas de $2 \mathrm{~m}$. Não foi admitida uma força do solo agindo sobre a esfera, com valores menores que zero, assim como a força de corte da cultura foi desprezada. Para avaliar o desvio entre os valores de força de contato simulada e a força de contato de referência do controlador, utilizou-se o erro relativo médio:

$$
e=100 \frac{\sum\left[\frac{\left|F_{C}-F_{R}\right|}{F_{R}}\right]}{n}
$$

em que:

e - erro relativo médio, \%

$\mathrm{n}$ - número de pontos simulados em cada tratamento

\section{RESULTADOS E DISCUSSÃO}

O controlador proporcional-integral-derivativo e as constantes $\mathrm{K}_{\mathrm{P}}$ igual a $1,0 \times 10^{-4} \mathrm{~m} \mathrm{~N}^{-1}, \mathrm{~K}_{\mathrm{i}}$ igual a $1,0 \times 10^{-6} \mathrm{~m} \mathrm{~N}^{-1} \mathrm{~s}^{-1} \mathrm{e}$ $\mathrm{K}_{\mathrm{d}}$ igual a $1,0 \times 10^{-5} \mathrm{~m} \mathrm{~s} \mathrm{~N}^{-1}$ foram as que ofereceram a combinação mais adequada de tempo de resposta e estabilidade do sistema. Em todas as simulações, os maiores erros se referem aos primeiros incrementos de simulação, fato atribuído às con- dições iniciais adotadas na solução do sistema de equações diferenciais, utilizando-se o método de Runge Kutta de $4^{\mathrm{a}}$ ordem.

As Figuras 3 e 4 apresentam o comportamento da posição da ponta esférica $\left(\mathrm{y}_{\mathrm{F}}\right)$, do perfil do solo $\left(\mathrm{y}_{\mathrm{s}}\right)$, do deslocamento do carretel da válvula de controle e do pistão e, também, as pressões na câmara de avanço $\left(\mathrm{P}_{1}\right)$ e de retrocesso $\left(\mathrm{P}_{2}\right)$. O comportamento desses parâmetros foi semelhante, em todas as simulações realizadas, mudando apenas sua magnitude, motivo pelo qual os parâmetros se apresentam característicos de uma situação escolhida ao acaso.

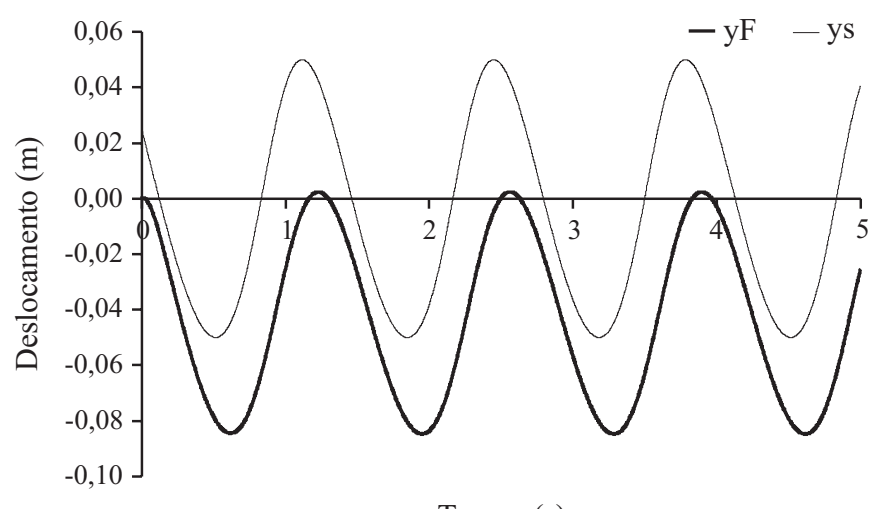

Tempo (s)

Figura 3. Deslocamento da ponta esférica da célula de carga relativa ao perfil do solo para velocidade de 1,5 $\mathrm{m} \mathrm{s}^{-1}$, pressão de 6,8 MPa, lastro de $80 \mathrm{~kg}$ e força de referência de $400 \mathrm{~N}$

O deslocamento da ponta esférica da célula de carga foi idêntico àquele do perfil do solo, embora se tenha verificado pequena defasagem de $0,11 \mathrm{~s}$ entre eles. Este comportamento pode ser observado na Figura 3, em que a defasagem entre o deslocamento dos perfis indica que a penetração da ponta esférica é variável. Quando a plataforma descia ocorria menor penetração da ponta esférica $(\mathrm{yF})$, enquanto na subida a tendência foi de aumento do enterramento.

Nos intervalos em que o deslocamento da válvula se apresenta com valores negativos, acontece a diminuição nos valores de deslocamento do pistão, Figura 4, provocado pelo aumento do orifício de passagem do fluido hidráulico até a câmara de retrocesso. Nesta situação, o pistão se retrai e faz descer a plataforma de corte; caso contrário, quando o deslocamento da

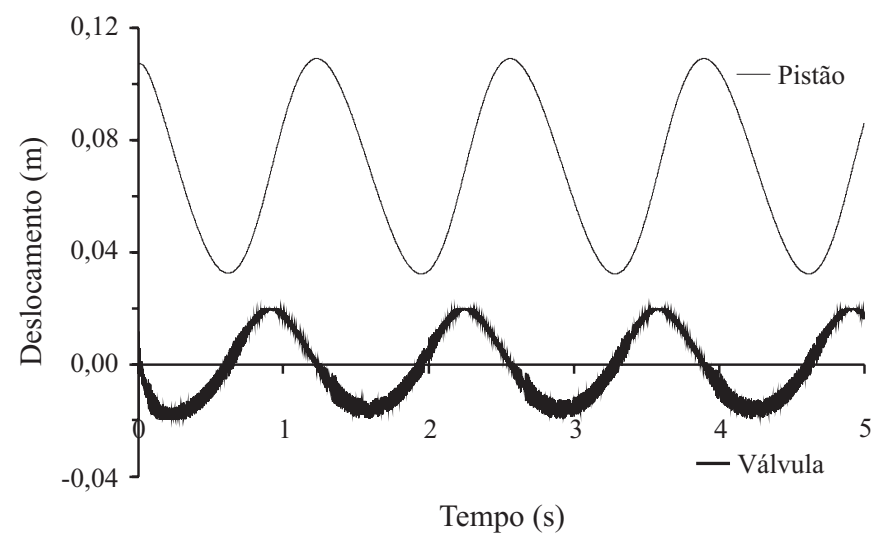

Figura 4. Deslocamento do carretel da válvula e do pistão para velocidade de $1,5 \mathrm{~m} \mathrm{~s}^{-1}$, pressão de $6,8 \mathrm{MPa}$, lastro de $80 \mathrm{~kg}$ e força de referência de $400 \mathrm{~N}$ 
válvula se apresenta positivo, indica a passagem do fluido até a câmara de avanço e o conseqüente prolongamento do pistão, representado pelo aumento na sua curva de deslocamento. Apesar do pistão hidráulico ter um curso máximo de $0,20 \mathrm{~m}$, foram suficientes $0,0768 \mathrm{~m}$ para corrigir os sinais de erro apresentados neste experimento de simulação; para tal situação, é necessário que o carretel da válvula se desloque até os limites máximos $(0,020 \mathrm{~m})$ e mínimos $(-0,020 \mathrm{~m})$ para minimizar o sinal de erro.

Analisando-se, na Figura 5, o comportamento das pressões nas câmaras de avanço e retrocesso, verifica-se que a pressão máxima na câmara de avanço da plataforma $\left(\mathrm{P}_{1}\right)$

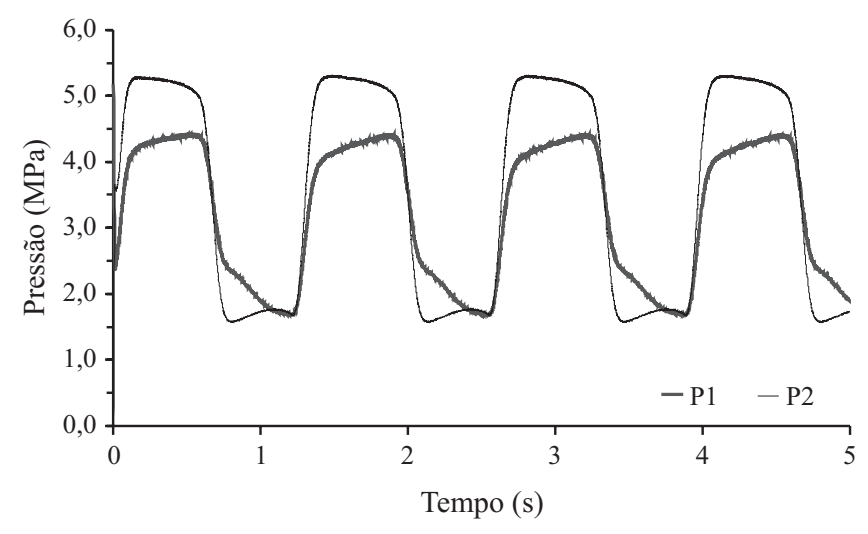

Figura 5. Pressão na câmara de levantamento $\left(P_{1}\right)$ e na câmara de abaixamento $\left(\mathrm{P}_{2}\right)$ para velocidade de $1,5 \mathrm{~m} \mathrm{~s}^{-1}$, pressão de $6,8 \mathrm{MPa}$, lastro de $80 \mathrm{~kg}$ e força de referência de $400 \mathrm{~N}$ representa $66 \%$ da pressão de suprimento do sistema hidráulico, enquanto a pressão máxima na câmara de retrocesso da plataforma $\left(\mathrm{P}_{2}\right)$ representa $80 \%$. Concluiu-se que os valores máximos de $\mathrm{P}_{1}$ são menores que os máximos de $\mathrm{P}_{2}$, conseqüência da diferença de áreas do pistão do atuador, sobre as quais atua o fluido hidráulico. No caso em que $\mathrm{P}_{1}$ aumenta, significa que o fluido está passando para a câmara que favorece o levantamento da plataforma e, no mesmo instante, o fluido contido na câmara de retração retorna ao depósito de óleo.

Notou-se que a força de contato tendeu a se igualar à força de referência do controlador com o aumento do tempo, para as pressões de 13,7 e 20,6 MPa, independentemente da velocidade de deslocamento do veículo. Na pressão de 6,8 $\mathrm{MPa}$ e velocidade de $2,0 \mathrm{~m} \mathrm{~s}^{-1}$, a força de contato apresentou comportamento diferente das outras duas velocidades, Figura 6, caso em que o controlador conseguiu apenas igualar a força de contato medida pelo transdutor com a força de referência, durante breves intervalos. Este comportamento, por sua vez, indica a incapacidade do controlador em corrigir o sinal de erro para essas condições.

Nas simulações realizadas para a pressão de 6,8 MPa e velocidades de 1,0 e $1,5 \mathrm{~m} \mathrm{~s}^{-1}$, a força de contato tendeu a um valor constante, mostrando que o controlador funcionaria satisfatoriamente nessas condições. Analisando-se os erros relativos médios das forças de contato, apresentados na Figura 6, verificou-se que para a pressão de 6,8 MPa, os valores foram semelhantes e inferiores a $0,27 \%$, utilizandose velocidades de 1,0 e $1,5 \mathrm{~m} \mathrm{~s}^{-1}$, enquanto na velocidade de

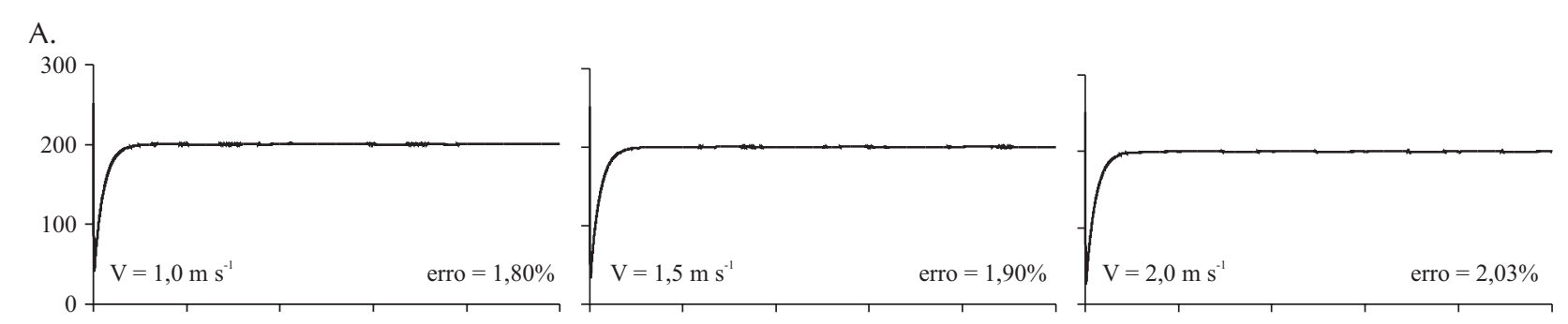

B.

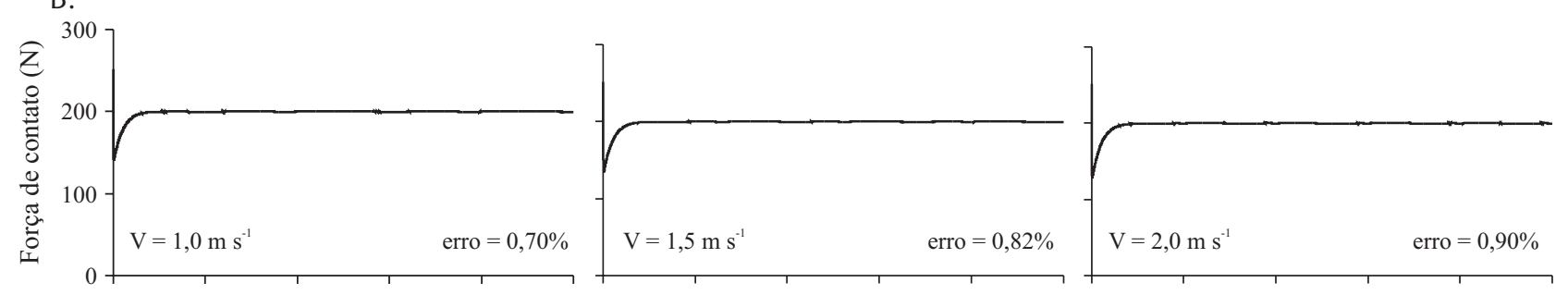

C.

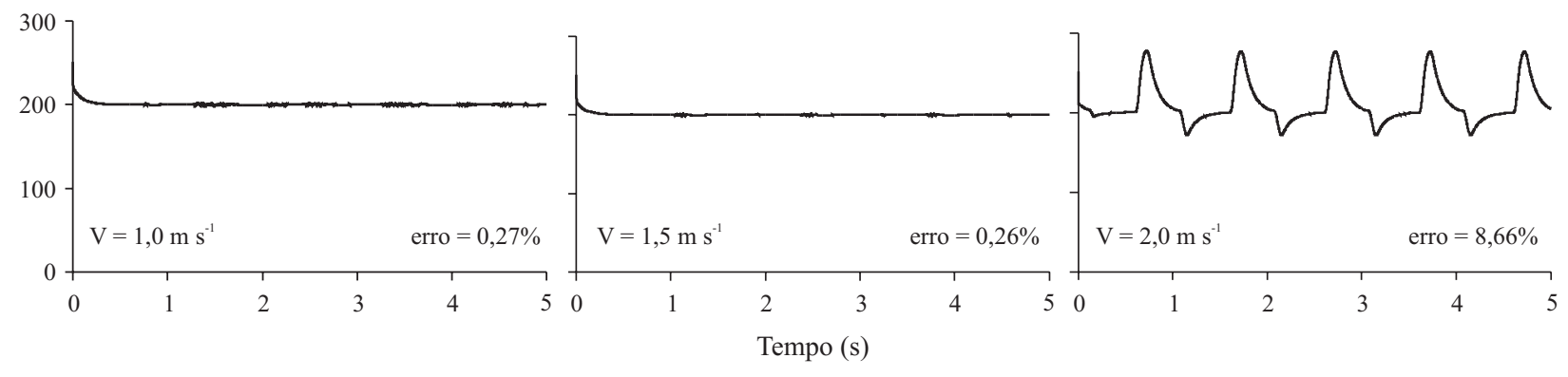

Figura 6. Força de contato em função do tempo para as respectivas velocidades, com pressões de 20,6 (A), 13,7 (B) e 6,8 MPa (C), lastro de 80 kg e força de referência de $200 \mathrm{~N}$ 
2,0 $\mathrm{m} \mathrm{s}^{-1}$, eles ascenderam 8,66\%. Para os experimentos realizados com as pressões de 13,7 e 20,6 MPa, os erros relativos médios se mantiveram menores que $1,90 \%$, sendo que para a pressão de 20,6 MPa foram observados maiores valores de erro que para a pressão de 13,7 MPa. Os erros no controle da altura de corte foram considerados satisfatórios na maioria das simulações, exceto para a velocidade de deslocamento de 2,0 $\mathrm{m} \mathrm{s}^{-1}$ e pressão de 6,8 MPa.

Analisando-se as velocidades e pressão de suprimento, quando as simulações foram realizadas para as forças de contato de referência de 400 e $800 \mathrm{~N}$ e lastro da plataforma de 40 e $60 \mathrm{~kg}$, notou-se que a força de contato simulada apresentou comportamento semelhante àquele das apresentadas na Figura 6.

Em relação à influência do lastro da plataforma no comportamento do sistema, verificou-se, em todos os casos estudados que, a medida em que a massa de lastros aumenta, a capacidade do controlador de corrigir o erro melhora, independentemente da pressão de suprimento do sistema, da velocidade e da força de contato de referência (Tabela 3). A ação do peso da plataforma faz com que a velocidade angular da barra articulada aumente quando o atuador tem que realizar a ação de descida, indicando que o peso contribui para melhorar o desempenho do sistema.

Embora os dados simulados tenham apresentado comportamento semelhante para as três forças de contato de referências observa-se, na Tabela 3, que a força de $400 \mathrm{~N}$ proporcionou menores valores de erro relativo médio.

Tabela 3. Erro relativo médio da força de contato (\%) em função do lastro da plataforma e da força de referência

\begin{tabular}{cccc}
\hline \multirow{2}{*}{ Força de Referência $(\mathbf{N})$} & \multicolumn{4}{c}{ Lastro da Plataforma $\mathbf{( k g )}$} \\
\cline { 2 - 4 } & $\mathbf{4 0}$ & $\mathbf{6 0}$ & $\mathbf{8 0}$ \\
200 & 3,14 & 2,45 & 1,93 \\
400 & 2,27 & 2,08 & 1,92 \\
800 & 3,68 & 3,33 & 2,96 \\
\hline
\end{tabular}

Desta maneira e se analisando os resultados de todos os experimentos de simulação realizados com o sistema de controle verificou-se, independentemente do lastro e da força de referência usada nos experimentos de simulação, que nas situações em que coincidiam a menor pressão de suprimento $(6,8 \mathrm{MPa})$ e a maior velocidade de deslocamento $\left(2,0 \mathrm{~m} \mathrm{~s}^{-1}\right)$, a força de contato se tornava muito instável, indicando a incapacidade do controlador de corrigir o erro favorecendo, portanto, o ineficiente acompanhamento da superfície do solo, significando que o sistema hidráulico deveria trabalhar com uma pressão de, no mínimo, 13,7 MPa, e que existe uma pressão mínima para o sistema se comportar de maneira estável.

\section{CONCLUSÕES}

1. O sistema simulado proposto para controlar a posição da plataforma de corte é capaz de copiar um contorno senoidal do solo, com eficiência.

2. A força de contato não foi influenciada pela velocidade de deslocamento a maiores pressões.

3. O aumento na pressão de suprimento e na massa da plataforma proporcionou melhores condições para o funcionamento do sistema de controle.

4. Os menores valores de erro relativo médio ocorreram na força de referência de $400 \mathrm{~N}$.

\section{AGRADECIMENTOS}

À CAPES - Coordenação de Aperfeiçoamento de Pessoal de Nível Superior, e ao CNPq - Conselho Nacional de Desenvolvimento Científico e Tecnológico, pelas bolsas de estudo concedidas.

\section{LITERATURA CITADA}

Gómez, A. Sistema cubano de cosecha en verde. In: Conferencia Científica Internacional, 1996, Ciudad Habana. Resumenes... Ciudad Habana: Agromac, 1996. CD Rom

Lopes, G. T. Controle ótimo de altura da plataforma de corte de colhedoras automotrizes. Campinas: UNICAMP, 2000. 134p. Tese Doutorado

MDI. ADAMS/Solver reference manual. Ann Arbor: Mecanical Dynamics Inc. 1993. CD Rom

Merritt, H. E. Hydraulic control systems. 1.ed. New York: John Wiley \& Sons, 1967. 358p.

Ogata, K. Engenharia de controle moderno. Rio de Janeiro: Prentice/Hall do Brasil, 1985. 929p.

Press W.H.; Teukolsky, C. S. A.; Vetterling, W.; Flannery, B. P. Numerical recipes in Fortran. Second Edition. Cambridge: Cambridge University Press. 1992. 963p.

Rafull, L. Z. L.; Souza, C. M. A.; Queiroz, D. M. Una revisión sobre el control automático de la altura de corte en cosechadoras. Revista Ciencias Técnicas Agropecuarias. Habana, v.13, n.3, p.29-35. 2004.

Souza, C. M. A.; Queiroz, D. M.; Cecon, P. R.; Mantovani, E. C. Avaliação de perdas em uma colhedora de fluxo axial para feijão. Revista Brasileira de Engenharia Agrícola e Ambiental, Campina Grande, v.5, n.3, p.530-537. 2001.

Winston, W. L. Operations research: applications and algorithms. 3.ed. Belmont: Wadsworth Publishing Company, 1994. 1353p. 